\title{
Gibt es eine neue Kopfspeicheldrüse? - Eher nicht!
}

\section{Is there a new salivary gland? - Rather not!}

\author{
Autoren \\ Friedrich Paulsen ${ }^{5}$, Andreas Dietz ${ }^{7}$, Ingo Bechmann ${ }^{8}$ \\ Institute \\ 1 Klinik und Poliklinik für Hals-Nasen-Ohrenheilkunde, \\ Universitätsklinikum Jena \\ 2 Labor für Dermatohistologie und Oralpathologie, \\ München \\ 3 Klinik für Nuklearmedizin, Universitätsklinikum Jena \\ 4 Klinik und Poliklinik für Nuklearmedizin, \\ Universitätsklinikum Leipzig \\ 5 Institut für Funktionelle und Klinische Anatomie, \\ Friedrich-Alexander-Universität Erlangen \\ 6 Hals-, Nasen- und Ohrenklinik, Kopf- und Halschirurgie, \\ Universitätsklinikum Erlangen \\ 7 Klinik für Hals-Nasen-Ohrenheilkunde, \\ Kopf- und Halschirurgie, Universitätsklinikum Leipzig \\ 8 Institut für Anatomie, Universität Leipzig \\ online publiziert 16.11 .2020 \\ Bibliografie \\ Laryngo-Rhino-Otol 2021; 100: 12-14 \\ DOI 10.1055/a-1307-3872 \\ ISSN 0935-8943 \\ (c) 2020. Thieme. All rights reserved. \\ Georg Thieme Verlag KG, Rüdigerstraße 14, \\ 70469 Stuttgart, Germany \\ Korrespondenzadresse \\ Univ.-Prof. Dr. med. Orlando Guntinas-Lichius \\ Klinik und Poliklinik für Hals-Nasen-Ohrenheilkunde \\ Universitätsklinikum Jena, Am Klinikum 1, 07747 Jena, \\ Deutschland \\ Tel.: +49/3641/9329301 \\ Fax: $+49 / 3641 / 9329302$ \\ orlando.guntinas@med.uni-jena.de
}

Orlando Guntinas-Lichius ${ }^{1}$, Stephan Ihrler², Martin Freesmeyer ${ }^{3}$, Falk Gühne ${ }^{3}$, Regine Kluge ${ }^{4}$, Lars Bräuer ${ }^{5}$, Heinrich Iro ${ }^{6}$,

\section{ZUSAMMENFASSUNG}

Im Oktober 2020 vermeldeten die Laienpresse, aber auch einige medizinische Journale und Internetseiten, die angebliche Entdeckung einer neuen Speicheldrüse im Nasenrachen ausgehend von Positronen-Emissions-Tomografie-Computertomografie-Untersuchungen mit Liganden für das Prostata-spezifische Membranantigen (PSMA-PET/CT). Als interdisziplinäre Gruppe aus den Fachbereichen Anatomie, Pathologie, Nuklearmedizin und HNO-Heilkunde kommen wir zu der Ansicht, dass hier eine Anhäufung von kleinen Speicheldrüsen beschrieben wird. Kleine Speicheldrüsen im Nasenrachen und peritubar sind mindestens seit 1866 bekannt und immer wieder in der Literatur und in Lehrbüchern beschrieben. Das PSMA-PET/CT könnte aber geeignet sein, bei einer Bestrahlungsplanung funktionell relevante Anhäufungen von Schleimhautspeicheldrüsen, wie beispielsweise im Bereich des Nasenrachens, besser darzustellen und zu schonen. Dies sollte in klinischen Studien geprüft werden.

\section{ABSTRACT}

In October 2020, the lay press, but also some medical journals and websites reported the putative discovery of a new salivary gland in the nasopharynx based on prostate-specific membrane antigen positron emission tomography computed tomography (PSMA-PET/CT) examinations. As an interdisciplinary group from the fields of anatomy, pathology, nuclear medicine and otorhinolaryngology, we come to the view that an accumulation of minor salivary glands has been described here. Minor salivary glands in the nasopharynx and in the peritubar region have been described at least since 1866 . The current description in PSMA-PET/CT does not justify the definition of a new, independent salivary gland. The PSMA-PET/CT could, however, be suitable to better protect salivary glands in the nasopharynx when planning radiation therapy. This should be evaluated in clinical trials.

\section{Mit einem ${ }^{68} \mathrm{Gallium}-\mathrm{PSMA}-\mathrm{PET} / \mathrm{CT}$ lassen sich Kopfspeicheldrüsen darstellen - warum auf einmal die mediale Aufmerksamkeit?}

Niederländische Kollegen um Mathijs H. Valstar vom Netherlands Cancer Institute in Amsterdam berichten in der Fachzeitschrift
Radiotherapy and Oncology unter dem Titel „The tubarial salivary glands: A potential new organ at risk for radiotherapy“ [1]. Bei der Analyse von ${ }^{68} \mathrm{Gallium}-\mathrm{PSMA}-\mathrm{PET} / \mathrm{CT}$-Untersuchungen ist den Autoren aufgefallen, dass der Tracer außer den großen Kopfspeicheldrüsen auch regelmäßig eine Region im Nasenrachen direkt neben dem Torus tubarius beidseitig markierte. Dies mag die Pu- 
blikation in einem radiotherapeutischen Journal und nicht etwa in einem anatomischen Journal erklären. PSMA steht für Prostataspezifisches Membranantigen (prostate-specific membrane antigen). Die ${ }^{68}$ Gallium-PSMA-PET/CT wird seit einigen Jahren in der Diagnostik von Prostatakarzinomen eingesetzt [2]. Die niederländische Arbeitsgruppe hat bereits 2018 ausführlich die physiologische PSMA-Expression im ${ }^{68}$ Gallium-PSMA-PET/CT auch in gesunden großen und kleinen Speicheldrüsen sowie in der Tränendrüse beschrieben [3]. So ist das ${ }^{68} \mathrm{Gallium}-\mathrm{PSMA}$-PET/CT auch geeignet, eine akzessorische Gl. parotis zu detektieren [4]. Die lokale Mehranreicherung in der exemplarischen Falldarstellung in der neuen niederländischen Publikation zeigt einen lokal erhöhten PSMAUptake als Ausdruck einer dichten Anhäufung von Drüsen. Dies wäre aber durchaus mit einer lokalen Akkumulation einzelner Speicheldrüsen erklärbar. ${ }^{68}$ Gallium-PSMA-PET/CT-bildmorphologisch liegt hier nicht notwendigerweise ein neues Organ vor und somit auch kein Beweis für ein mögliches neues, bislang nicht beschriebenes eigenes Organ.

In der Arbeit der niederländischen Kollegen wurde histologisch an nur 2 Körperspendern Speicheldrüsengewebe in der Schleimhaut am Torus tubarius nachgewiesen, das sich korrelierend zu den Beobachtungen in der ${ }^{68}$ Gallium-PSMA-PET/CT immunhistochemisch mit PSMA markieren ließ. Die Kollegen sind der Ansicht, eine neue Kopfspeicheldrüse, die „Tubendrüse“, entdeckt zu haben, was sich jedoch mit den beschriebenen Befunden nicht belegen lässt. Dass hier tatsächlich ein neues Organ entdeckt wurde, ist somit eher eine mediale Sensation, als dass wissenschaftlich wesentliche neue, anatomisch-relevante Erkenntnisse über den Menschen gemacht wurden.

\section{Auch im Nasenrachen sind kleine Speicheldrüsen lange bekannt}

Ca. 800-1000 kleine Speicheldrüsen sind im Bereich der Schleimhaut von Lippen, Mundhöhle, Pharynx einschließlich Nasopharynx, Nase und Nasennebenhöhlen, Larynx, Trachea, Tuba auditiva und Mittelohr in unterschiedlicher Dichte verteilt. Sie sind in der anatomischen Literatur im Nasopharynx gut beschrieben [5, 6]. Kleine Speicheldrüsen in der Nasopharynx- und Tubenschleimhaut wurden bereits in den anatomischen Lehrbüchern von Henle (1866) und Kopsch (1909) als Schleimdrüsen ausführlich aufgeführt $[7,8]$. In moderneren Anatomiebüchern findet man Abbildungen von den Drüsen um die Tube; sie werden als Glandulae tubariae beschrieben, aber ebenso wie die Drüsen etwa des Gaumens nicht als eigenständiges Organ aufgefasst. Anton von Tröltsch benutzte den Begriff der Tubendrüsen für die Beschreibung von Drüsen nahe des Paukenhöhlenostiums (zitiert in: [7]).

Kleine Speicheldrüsen sind in der Regel nicht makroskopisch sichtbar. Nur an den Lippen, wie es HNO-Ärzte von der SjögrenDiagnostik kennen, kann man makroskopisch einzelne Drüsen auf der Lippeninnenseite erahnen. Auch eine konventionelle Bildgebung ist nicht in der Lage, diese Drüsen darzustellen. Auffällig werden diese kleinen Speicheldrüsen nur im Fall einer Erkrankung, sei es bei einem Speicheldrüsentumor, einer Retentionszyste oder einer Extravasationsmukozele. Beide, Tumoren und Zysten, kommen noch relativ häufig in der Mundhöhle und hier vor allem am Gaumen mit einer bekannt hohen Dichte an kleinen Speicheldrüsen vor; im Nasenrachen treten sie hingegen nur äußerst selten auf $[9,10]$. In einer eigenen aktuellen Untersuchung (Ihrler et al., nicht publizierte Daten) finden sich nur 8 von 378 Tumoren kleiner Speicheldrüsen $(2,1 \%)$ im Nasenrachen, jedoch z. B. $47,6 \%$ am Gaumen. Zahlen zur Häufigkeit von Tumoren kleiner Speicheldrüsen im Nasenrachen sind überraschenderweise in der Literatur nicht existent.

\section{Es spricht klar mehr dagegen als dafür, dass eine neue Kopfspeicheldrüse entdeckt wurde}

Wenn die mittels ${ }^{68}$ Gallium-PSMA-PET/CT dargestellte Anhäufung kleiner Speicheldrüsen in der Umgebung der Tube ein eigenständiges Organ darstellen sollte, würde man statistisch wesentlich mehr Erkrankungen erwarten. Die histologischen Abbildungen in der niederländischen Publikation (dort als Abb. 3) zeigen das typische Bild der bekannten kleinen Speicheldrüsen entsprechend der bestehenden anatomischen und pathologischen Erfahrung [11], aber kein mit den großen Speicheldrüsen vergleichbares eigenständig abgekapseltes Organ.

Alle Überlegungen sprechen zusammenfassend dafür, dass in der vorliegenden Publikation lediglich eine dichtere peritubare Anhäufung von histologisch regelhaften und in der anatomischen, pathologischen und klinischen Literatur gut bekannten kleinen Speicheldrüsen mit dem PSMA-PET/CT dargestellt wurde. Ausreichende Kriterien zur Annahme eines „neuen Organs“ sehen wir damit jedoch nicht. Selbstverständlich sollte auch diese Gruppe kleiner Speicheldrüsen - wie jedes Speicheldrüsengewebe - möglichst bei einer Bestrahlung geschont werden.

\section{FAZIT FÜR DIE PRAXIS}

- Die PSMA-PET/CT ist gut geeignet, um Speicheldrüsengewebe darzustellen.

- Es ist seit langem bekannt, dass sich auch im Nasenrachen und peritubar kleine Speicheldrüsen finden.

- Eine intensivere PSMA-PET/CT-Darstellung von Speicheldrüsengewebe peritubar spricht für eine Anhäufung von kleinen Speicheldrüsen, aber nicht für ein eigenständiges neues Organ.

- Bei einer Bestrahlungsplanung könnte die PSMA-PET/CT genutzt werden, um gezielt die Bestrahlung von Speicheldrüsengewebe zu vermeiden. Dies sollte in klinischen Studien evaluiert werden.

Interessenkonflikt

Die Autorinnen/Autoren geben an, dass kein Interessenkonflikt besteht. 


\section{Literatur}

[1] Valstar MH, de Bakker BS, Steenbakkers R et al. The tubarial salivary glands: A potential new organ at risk for radiotherapy. Radiother Oncol 2020. doi:10.1016/j.radonc.2020.09.034

[2] Afshar-Oromieh A, Hetzheim H, Kratochwil C et al. The Theranostic PSMA Ligand PSMA-617 in the Diagnosis of Prostate Cancer by PET/CT: Biodistribution in Humans, Radiation Dosimetry, and First Evaluation of Tumor Lesions. J Nuclear Med o 2015; 56: 1697-1705

[3] Klein Nulent TJW, Valstar MH, de Keizer B et al. Physiologic distribution of PSMA-ligand in salivary glands and seromucous glands of the head and neck on PET/CT. Oral Surg Oral Med Oral Pathol Oral Radiol 2018; 125 : 478-486

[4] Guhne F, Drescher R, Aschenbach R et al. Dedicated Verification of an Accessory Parotid Gland via Minimal-Activity PSMA-PET/CT. Tomography 2020; 6: 288-289
[5] Tillmann B. Farbatlas der Anatomie Zahnmedizin - Humanmedizin. 1. Aufl. Stuttgart: Georg Thieme; 1997

[6] Lippert H. Lehrbuch Anatomie. 7. Aufl. München: Elsevier - Urban \& Fischer; 2006

[7] Henle J. Handbuch der systematischen Anatomie des Menschen. Braunschweig: Friedrich Vieweg und Sohn; 1866

[8] Kopsch F. Rauber's Lehrbuch der Anatomie des Menschen. 8. Aufl. Leipzig: Georg Thieme; 1909

[9] Guzzo M, Locati LD, Prott F] et al. Major and minor salivary gland tumors. Cri Rev Oncol Hematol 2010; 74: 134-148

[10] Zhang XM, Cao JZ, Luo JW et al. Nasopharyngeal mucoepidermoid carcinoma: a review of 13 cases. Oral Oncol 2010; 46: 618-621

[11] Pires FR, Pringle GA, de Almeida OP et al. Intra-oral minor salivary gland tumors: a clinicopathological study of 546 cases. Oral Oncol 2007; 43: $463-470$ 